\title{
Industrial robot ethics: facing the challenges of human-robot collaboration in future manufacturing systems
}

\author{
S.R Fletcher and P. Webb \\ Centre for Advanced Systems, Cranfield University, UK \\ email: s.fletcher@cranfield.ac.uk
}

\begin{abstract}
As a result of significant advances in information and communications technology the manufacturing industry is facing revolutionary changes whereby production processes will become increasingly digitised and interconnected cyber-physical systems. A key component of these new complex systems will be intelligent automation and augmented human-robot collaboration. Industrial robots have traditionally been segregated from people in manufacturing systems because of the dangers posed by their operational speeds and heavy payloads. However, considerable developments in intelligent automation means we will soon see autonomous smallscale robotics and safe large-scale robots being used to work more closely and collaboratively with people as part of advanced manufacturing systems. This will not only transform the way people are expected to work and interact with the automation on production tasks but will also involve much more data provision and capture for performance monitoring. This paper describes anticipated issues in further development and implementation of industrial robots that will be able to work collaboratively with human operators and the associated ethical issues that need to be addressed.
\end{abstract}

Keywords: manufacturing, industrial robots, human-robot collaboration.

\section{INTRODUCTION}

It is commonly accepted that we are now at an early stage of a fourth industrial revolution, 'Industrie 4.0', in which shop floor environments will be transformed into more automated, integrated and digitally inter-connected cyberphysical systems (CPS) [1]. Advances in the development of computer mediated communications and information technology will not only enable adaptive control of systems but multifarious intelligent interactions between physical and autonomous elements, such as robots [2]. To enhance competitiveness and flexibility, this development of more intelligent systems and 'cloud manufacturing' will convert traditional sequential man-machine production processes into more sophisticated assemblages of highly digitised networks which not only communicate internally but also with other systems and organisations externally [3]. Systems will involve greater integration of humans and automation via various components such as informatics, robotics, mobile devices and sensors. Although it is claimed that "Industrie 4.0 is not initiated on a shop floor level" [4] there is no denying that the conventional shopfloor environments will be transformed.
Despite long industrial aspirations for fully automated 'lights out' systems with no direct human input it is now clear that manufacturing systems will continue to rely on humans on the shop floor for decision making and dexterity in assembly tasks [5] and increasingly for the supervision and maintenance of technical systems and machines [6]. Traditionally, issues affecting human operators have not been considered sufficiently in the design or redesign of industrial systems despite how significantly human issues and behaviour has been recognised as a major influence on overall system performance [7]. It has long been noted that insufficient consideration of human issues is a root cause of unsuccessful implementation of advanced manufacturing technologies [8]. Although better integration of human factors into the engineering design of industrial systems and work processes is steadily gaining more importance [9] it typically remains a little out of step with the level of attention that is customarily given to developing technical capability. It is reasonable to assume, therefore, that attention to human ethical issues in relation to new more intelligent, interconnected and cyberphysical systems is also likely to be relatively neglected. 
Advancing technologies in every area of life mean that humans will need to adapt to increasing levels of intelligent automation, informatics and CPS in their daily protocols and not just at work. However, this paper specifically addresses the impending ethical issues that will inevitably be brought about by the Industrie 4.0 revolution in manufacturing work and focuses particularly on one crucial aspect: the impending escalation of automation and collaborative human-robot systems.

\section{INDUSTRIAL HUMAN-ROBOT COLLABORATION DEVELOPMENT}

As discussed above, it is expected that future production systems will still rely on work from both humans and automation albeit in new and different arrangements. However, there has been a steady trend over many years of manufacturing organisations seeking to increase levels of automation to improve their competitive advantage, and this is likely to continue as industrial automation will inevitably play a critical role in future CPS and 'cloud manufacturing' systems [10]. The problem is that although generally there is better recognition of the importance of human issues, the higher levels of automation in modern production systems have "already been taken far without paying sufficient attention to the specific knowledge, skills and abilities of the human operator" [11]. Thus, there is a need to consider the likely trajectory and impacts of enlarging levels of automation on the human workforce.

To date, people have had to be segregated from medium and large scale industrial robots to protect them from the potential hazards posed by their payload strength and speed. To comply with previous health and safety requirements, industrial robots have not only always been segregated from operators in terms of functional position in the production system but by the use of salient physical guarding / 'caging' and safe distance measures. These robots are usually employed upstream in manufacturing systems in order to apply their superior strength and reliability on simple unskilled operations, typically at fully automated stations [12], whilst operators are positioned to apply dextrous and cognitive skills on more complex assembly tasks further downstream [13]. Thus, due to this traditional arrangement, many industrial workforces are already psychologically primed to expect segregation and mistrust proximity to robots on the shop floor. The problem is this segregation of human and robot work tends to reduces work flow efficiency, and it would be preferable if they could be enabled to work more closely together at points in the systems which involve skilled and nonskilled tasks. Thus, the concept of human-robot collaboration refers to the desirable real time union of human and robot in a shared workspace such that production activities are continuous and uninterrupted.

It is becoming increasingly possible to produce systems which will combine advanced control algorithms, metrology and sensory systems to enable humans and robots to work in closer proximity safely but without the same degree of restricted activity [14]. Industry also now has greater availability of force/torque limited robots which mean that physical contact is permitted and accepted if impact is mild enough not to cause harm. However, despite the development of a "solid science base" of human factors knowledge in relation to automation over many years [15] it has been observed that "engineers who develop robots" still tend to lack understanding and / or application of relevant psychological principles such as cognition and perception [16]. This implies that the development of new manufacturing systems may follow the traditional pitfalls of not sufficiently considering human issues.

Ongoing research in the UK has made significant progress in establishing the proof of concept for an exemplar large scale human-robot collaborative work system which combines commercially available robots and electro-sensitive protective all-round $\left(360^{\circ}\right.$ and topographical) vision safety monitoring system to create a wholly monitored environment which theoretically should be safer than traditional physical guarding. This programme is progressing, with experimental work moving steadily further towards the stage of shop-floor implementation and integration [17]. Importantly, as part of this programme of work, parallel studies are being conducted to address various human issues. For example, one body of this work is devoted to a comprehensive review of existing international standards governing the design of industrial human-robot collaboration and to developing and verify key system safety features and risk acceptance [18]. Additionally, extensive work has identified key factors likely to influence organisational implementation of human-robot collaboration as well as the individual-level factors likely to affect workers' adoption of, and trust in, human-robot collaboration systems [19]. In addition to these various projects regarding medium and large scale robots it is also the case 
that small scale robots are also being developed for shop floor use; although these will serve as assistive tools they will still technically constitute human-robot collaboration in future digitised systems.

\section{ETHICAL CONSIDERATIONS}

The transformations that will be brought about by the development of 'Industrie 4.0' shop floor environments will be multifarious. Human operators will be required to adapt to a vast number of physical, psychological and social changes to their workplace environments and to the actual nature of the work they do. All of these changes will need to be considered and managed appropriately to ensure human factors and ethics are adequately addressed in the dual interests of supporting both the wellbeing of the workforce and the success of technological developments and productivity. In this paper we are focusing specifically on the potential ethical issues surrounding human-robot collaboration and the significant transformation of the human role that it will bring to many members of the global manufacturing workforce.

As discussed, there is now a wide confidence and acceptance that people will still be required to work within future manufacturing systems, despite increased levels of automation. Indeed, it is the very escalation of automation that will bring one of the biggest changes to the human role. Not only will a large number of operators now need to be retrained and upskilled to replace their manual production tasks with more supervisory and monitoring roles there will also be a requirement for many members of the workforce to work directly with industrial robots in shared-zone collaborative systems. So, it is important that ethical issues are considered with respect to the management of this significant change so that we can help operators move from traditional methods and manual activities to new supervisory control roles and requirements and optimise the success of new systems. Although there are a great many potential issues to be deliberated and discussed in this area the following sections of this paper now consider three anticipated key areas: protection from harm, informed choice and confidentiality and performance data monitoring; these are now discussed in turn.

\subsection{Protection from (psychological) harm}

Firstly, the new expectation for a human to even venture near to an industrial robot will require a new mind set and level of trust. Traditional physical measures to segregate industrial robots from operators will have established distinct and visible proximity boundaries which prevented contact, sending out an abundantly clear message to the surrounding workforce that the industrial robot is a danger and is to be avoided and kept at distance. This message will also have been reinforced via training, operational protocols and safety communications and requirements. Thus, although the monitoring capabilities of new human-robot collaboration systems means they should be inherently safer than traditional physical guarding measures, introducing the concept of human-robot collaboration will demand a completely new way of thinking which will require operators to change their long-standing traditions and attitudes towards robots.

Increasing availability of smaller and lighter force/torque limited robots which allow limited physical contact have already begun to change and challenge traditional attitudes to industrial applications of intelligent automation. Their safety is supported by various studies and reviews which have set acceptable levels of collision impact and injury. However, although implementation of these more 'harmless' systems allow greater humanrobot collaboration and worker compliance with CPS there is little in the literature to suggest that we know how workers themselves feel about the possibility of experiencing a 'safe' collision with one of these systems. Moreover, there appears to be little provision of information or communication exchange with workers with which we can determine the extent to which they are content with prescribed acceptable levels of collision impact and risk.

Additionally, future intelligent systems and robotics will inevitably comprise higher levels of autonomy and mobility on the shop floor, with advanced capabilities for self-learning and adaptation. These higher functions will obviously bring different impacts and demands to human operators, as they will not only have to accept that the system is adaptive but will also may be required to adapt their own behaviour in return. So again, although systems will undoubtedly be developed with sufficient technical and scientific robustness, and from this may be attributed with an acceptable safety rating, there is little evidence that we are addressing ethical issues surrounding potential psychological impacts and workers' education / acceptance. 
Ethically then, whereas physical guarding measures have been in place to prevent physical harm we now need to consider more the potential for psychological harm, as asking individuals to change their assumptions of danger or accept prescribed levels of risk acceptability may infuse various psychological concerns and anxieties. We cannot simply expect individuals to adopt a new concept of industrial robots that conflicts directly with what they have learned previously without supporting and educating them. Ethical considerations surrounding protection of individual operators must therefore be incorporated into training and remedial interventions within organisations - but to do this we need to first improve our understanding of potential impacts and relevant ethical issues.

As with all human-robot collaboration scenarios, these are going to bring significant changes to work practices and to optimise the chances of success they should not be imposed on workforces without good change management involving analysis of impacts linked to adjustment coping strategies.

\subsection{Informed choice and deception}

As outlined above, we need to consider and upscale our consideration of the changes that will be brought about by new 'Industrie 4' shop floor robotic systems. In order to foster a new outlook and sense of trust from operators it will be important to provide human-robot collaboration training with fairly comprehensive information about the functionality and reliability of the human-robot system so that they are fully aware of user protocols and levels of risk. Without enlightening potential users there will be a large risk of unsuccessful adoption and lack of trust development. Ethically, it is not only important to build up user trust in the human-robot systems but to do so based on the user being made aware of the facts and not via any form of misinformation or deception so that individuals are able to make an informed choice whether to accept the new role change.

Amongst the ethical issues concerning the introduction of such a new way of working and the requirements for attitude change / re-education that it will bring, there is also a question of voluntary redeployment and role change. Although workers must obviously comply with reasonable practices and procedures laid down by the organisation it would not be advantageous to force anyone to work in a system they do not trust or which causes them anxiety as this will also contribute to illness and therefore will not be in the interests of the worker or the organisation. Thus, such a significant change in work practices should not be imposed on workforces without analysis of the impacts with them linked to a provision of readjustment coping strategies. Additionally, an alternative issue would be how to manage the education and role change for an individual who perhaps does not mind taking on a new role in collaborative work with the robot but does not want to lose personal skills they have attained / built up over the years.

\subsection{Confidentiality and performance data monitoring}

A key feature of impending 'Industrie 4' manufacturing systems will be its inevitable collection and distribution of 'big data' via the various networks and interfaces between sensors, informatics, robotics and people. Collaborative human-robot sub-systems will no longer be designed to simply involve mechanistic joint 'turntaking' tasks, but will instead involve more complex interactions with other system elements and distributed systems of multiple robots and people. As part of the management of 'big data' systems these interactions will involve human data capture. It is inevitable that in many cases data will not just be delivered to the human-robot system but will also be collected from the human either deliberately as part of system performance monitoring or just as a by-product of the system's informatics inter-connectivity. This means that increased informatics and augmented reality capabilities of more highly digitised systems are likely to capture human data directly or indirectly, overtly or covertly. Obviously any data which could be linked to identifiable individuals should still need to comply with data protection protocols which would normally require subject awareness and permission. However, it is not yet clear how the systemic data capture of future systems will differ from current performance data management and therefore how it may need to be managed differently to maintain personal protection and ethical suitability.

Higher levels of data capture may or may not increase personal identification and scrutiny, but an important ethical issue to consider is how well we address human concerns about intrusion. Of course if we do foresee an inevitable increase in the level of personal monitoring and scrutiny this may constitute a considerable change which would require new protocols. Thus, the ethical issues surrounding the capture and management of data systems should be considered in advance. 


\section{CONCLUSION}

Robot ethics in relation to the impacts of forthcoming 'Industrie 4' manufacturing systems is a relatively new area for research and development. Ongoing research at Cranfield University has begun to explore the psychological impacts of industrial human-robot collaboration, including human trust, and the key organisational factors that are likely to influence implementation of collaborative systems [19, 20]. However, with the development of this body of knowledge still in its infancy the issues presented here are intended to raise awareness for future development and debate, alongside the progress of technological developments. Our motivation is that safe and ethical systems can be designed, and successfully implemented and adopted by manufacturing organisations and their human workforces.

\section{REFERENCES}

[1] Kagermann, H., Lukas, W., \& Wahlster, W. (2011). Industrie 4.0: Mit dem Internet der Dinge auf dem Weg zur 4. industriellen Revolution. VDI nachrichten, 13, 2011.

[2] Lanza, G., Haefner, B., \& Kraemer, A. (2015). Optimization of selective assembly and adaptive manufacturing by means of cyber-physical system based matching. CIRP Annals - Manufacturing Technology. In Press.

[3] Jacobs, R. (2015). Rise of Robot Factories Leading 'Fourth Industrial Revolution'.

Newsweek (US edition), March 5, 2015.

Accessed 21/06/15:

http://www.newsweek.com/2015/03/27/riserobot-factories-leading-fourth-industrialrevolution-311497.html

[4] Schuh, G., Reuter, C., Hauptvogel, A., \& Dölle, C. (2015). Hypotheses for a Theory of Production in the Context of Industrie 4.0. In Advances in Production Technology (pp. 1123). Springer International Publishing.

[5] Shen, Y., Reinhart, G., \& Tseng, M. M. (2015). A design approach for incorporating task coordination for human-robotcoexistence within assembly systems. In Systems Conference (SysCon), 2015 9th
Annual IEEE International (pp. 426-431). IEEE.

[6] The Economist (2012). Making the future: How robots and people team up to manufacture things in new ways. The Economist, April 21st, 2012. Accessed 30/05/15:

http://www.economist.com/node/2155289 

[7] Fletcher, S.R., Baines, T.S., \& Harrison, D.K. (2008). An investigation of production workers' performance variations and the potential impact of attitudes. International Journal of Advanced Manufacturing Technology, 35, 1113-1123

[8] Chung C.A. (1996). Human issues influencing the successful implementation of advanced manufacturing technology. Journal of Engineering and Technology Management, 13(3):283-299.

[9] Battini, D., Faccio, M., Persona, A., \& Sgarbossa, F. (2011). New methodological framework to improve productivity and ergonomics in assembly system design. International Journal of Industrial Ergonomics, 41(1), 30-42.

[10] Givehchi, O., Trsek, H., \& Jasperneite, J. (2013, September). Cloud computing for industrial automation systems-A comprehensive overview. In Emerging Technologies \& Factory Automation (ETFA), 2013 IEEE 18th Conference on (pp. 1-4). IEEE.

[11] Mayer, M. P., Schlick, C. M., Ewert, D., Behnen, D., Kuz, S., Odenthal, B., \& Kausch, B. (2011). Automation of robotic assembly processes on the basis of an architecture of human cognition. Production Engineering, 5(4), 423-431.

[12] Hedelind, M. \& Kock, S. (2011). Requirements on flexible robot systems for small parts assembly, a case study. Proceedings of the International Symposium on Assembly and Manufacturing, 25-27 May, Tampere, Finland, 2011. 
[13] De Krüger, J., Lien, T.K. \& Verl, A. (2009). Cooperation of human and machines in assembly lines. CIRP Annals Manufacturing Technology, 58, 628-646

[14] Santis, A., \& Siciliano, B. (2008). Safety issues for human-robot cooperation in manufacturing systems. Tools and Perspectives in Virtual Manufacturing.

[15] Parasuraman, R. \& Wickens, C.D. (2008). Humans: still vital after all these years of automation. Human Factors: The Journal of the Human Factors and Ergonomics Society, 50, 3, 511-520.

[16] Bartneck, C., Croft, E., \& Kulic, D. (2008). Measuring the anthropomorphism, animacy, likeability, perceived intelligence and perceived safety of robots. Proceedings of the Metrics for Human-Robot Interaction Workshop in affiliation with the 3rd ACM/IEEE International Conference on Human-Robot Interaction (HRI 2008), Technical Report 471, University of Hertfordshire, 37-44, Amsterdam.

[17] Walton M (2013). In the context of wing equipping - a framework for safeguarding direct cooperation between high load, industrial robots and human operators. Unpublished $\mathrm{PhD}$ Thesis, Cranfield University.

[18] Hamilton, A. \& Webb, P. (2015). Assessing the safety risk of collaborative automation within the UK aerospace manufacturing industry. Engineering Systems for Safety Proceedings of the Twenty-third SafetyCritical Systems Symposium, Bristol, UK. Safety-Critical Systems Club Publications

[19] Charalambous, G., Fletcher, S., \& Webb, P. (2015). Identifying the key organisational human factors for introducing human-robot collaboration in industry: an exploratory study. The International Journal of Advanced Manufacturing Technology, 1-13.

[20] Charalambous, G. (2014). The development of a human factors tool for the successful implementation of industrial human-robot collaboration. Unpublished $\mathrm{PhD}$ thesis, Cranfield University. 


\section{Industrial robot ethics: facing the challenges of human-robot collaboration in future manufacturing systems}

Fletcher, Sarah R.

Springer

S.R Fletcher and P. Webb. Industrial robot ethics: facing the challenges of human-robot collaboration in future manufacturing systems. Industrial Robot Ethics: The Challenges of Closer Human Collaboration in Future Manufacturing Systems, In: "A World with Robots: International Conference on Robot Ethics: ICRE 2015", 2017, pages 159-169 http://link.springer.com/chapter/10.1007/978-3-319-46667-5_12 Downloaded from Cranfield Library Services E-Repository 